\title{
Die Mischung macht's - Ein internationales Internetseminar
}

\author{
von Franziska Kühnemund, Rainer Nagel, Frank Räbiger, \\ Ulf Schlotterbeck und Roland Schnaubelt
}

Mit einem Workshop vom 18. bis 23. Juni 2000 am Heinrich Fabri-Institut in Blaubeuren endete das 3. TULKA ${ }^{1}$ Internetseminar Mathematik. Über 50 Teilnehmer aus 9 Ländern und allen 5 Kontinenten sind eindrucksvoller Beleg für das große internationale Interesse, das diese neue Seminarform gefunden hat, die eine Mischung aus virtuellem Seminar via Internetkommunikation und klassischem Seminar darstellt. Im folgenden stellen wir das Konzept des Seminars vor und berichten von unseren Erfahrungen bei seiner Realisierung.

Es gibt vielerlei Beweggründe, nun auch in der Mathematik auf der Multimedia- und Internetwelle mitzusurfen. Nicht unwichtig sind dabei der ImageGewinn bei Politik und Verwaltung sowie die oft großzügige finanzielle Förderung. Dass gerade diese Beweggründe aus der Sicht der reinen Wissenschaft oft als niedrig erscheinen, disqualifiziert leider den ganzen Bereich in den Augen vieler Kollegen. Ein Mathematiker surft nicht im Internet, kein Mausklick kann das Brüten über einem Problem ersetzen, kein Fenster auf dem Bildschirm die Nacht in der Bibliothek.

Dabei ist doch klar, dass sich vieles geändert hat. In keinem der Berufe, für die Staatsexamen oder Diplom in Mathematik direkt qualifizieren, akzeptiert man einen zweistündigen Monolog mit der Kreide in der Hand an der Tafel stehend. Vielmehr wird neben der fachlichen Kompetenz Erfahrung mit den neuen Medien, Teamfähigkeit und Internationalität gefordert. Insofern sollten die klassischen Seminarformen an unseren Hochschulen durchaus in Frage gestellt werden. Geht es nicht auch anders?

Wir haben einen Versuch gewagt und uns mit einem Internetseminar in das Verbundprojekt „Virtugrade“ (Virtuelle Graduiertenausbildung ) an der Universität Tübingen eingeklinkt. Nach nun drei Durchläufen $(1997 / 98,1998 / 99,1999 / 2000)$ ist es an der Zeit, über unsere Erfahrungen zu berichten.

Zunächst das Wichtigste vorweg: Der Erfolg des Projekts ist bemerkenswert und hat unsere Erwartungen weit übertroffen. Die Qualität der in den abschließenden Workshops präsentierten Vorträge war hervorragend, die Kontaktfreude, der Enthusiasmus und das Kommunikationsverhalten der Studenten einfach überwältigend. Wir wollen am Ende des Berichts versuchen, dies zu analysieren. Zuerst sollen aber Konzept und Ablauf des Seminars geschildert werden.

Das Internetseminar richtet sich in seiner jetzigen Form an Studenten ab dem dritten Studien- jahr mit Grundkenntnissen in Funktionalanalysis und Operatorentheorie. Die Themen der bisherigen Seminare waren "One-Parameter Semigroups and Evolution Equations" (1997/98), "Spectral Theory and Asymptotic Behavior of Semigroups" (1998/99) und "Semigroups Generated by Elliptic Operators" (1999/2000).

Die Integration des Seminarprojekts in den Verbund „Virtugrade“ an der Universität Tübingen, die fachliche Kooperation der an der Organisation beteiligten TULKA-Arbeitsgruppen in Tübingen (Prof. Dr. R. Nagel), Ulm (Prof. Dr. W. Arendt) und Karlsruhe (Prof. Dr. L. Weis) sowie die Unterstützung durch das Multimedia Labor Tübingen bieten die finanziellen, fachlichen und technischen Voraussetzungen zur Durchführung des Internetseminars (siehe Organisatorischer Rahmen).

Studying semigroups via internet was an intere-
sting addition to the courses offered at my univer-
sity.
(Petr Honzik, Prag)

Von zentraler Bedeutung sind außerdem die lokalen Koordinatoren: Jeder studentische Teilnehmer hat an seiner eigenen Universität einen (kompetenten) Ansprechpartner, der eine fachliche Bertreuung wie bei einem klassischen Seminar garantiert. Die "Rekrutierung" dieser Koordinatoren ist eine Grundvoraussetzung für das im folgenden vorgestellte Konzept.

\section{Das Dreiphasenmodell}

Das Internetseminar ist in drei Phasen gegliedert.

Phase 1 (Oktober-Februar). Nach einer zweiwöchigen Vorbereitungsphase beginnt das eigentliche Seminar. In wöchentlichem Abstand wird im BSCWWorkspace das Studienmaterial in kleinen Lernpaketen in Form von Postscript- und dvi-Dateien zur Verfügung gestellt. Hauptziel ist es hierbei, den Ausbildungsstand der Teilnehmer anzugleichen und das Ba-

1 TULKA steht für die Arbeitsgruppen Funktionalanalysis der Universitäten Tübingen, Ulm und Karlsruhe. 
siswissen zum Seminarthema zu vermitteln. Zur Förderung der Gruppendynamik und zur Vertiefung des Lernstoffes dienen Übungsaufgaben: Eine vom Organisationsteam benannte Gruppe von ca. 3-4 Studenten einer Universität löst gemeinsam die gestellten Aufgaben und gibt die Lösung über den Workspace allen Teilnehmern zur Ansicht. Sonderaktionen, wie z. B. wöchentliche online Sprechstunden mit dem Organisationsteam unterstützen die Zusammenarbeit. Zusätzlich gibt es im Workspace die Möglichkeit, Probleme zu diskutieren, um die Teilnehmer zur kontinuierlichen Mitarbeit anzuhalten.

Collaborating with a name, a virtual person, was a unique experience. Besides the interesting mathematics we shared and discussed, there was always the excitement of finding the 'real person' behind your virtual studying partner.

(Sachi Srivastava, Oxford)

Phase 2 (März-Juni) In Phase 2 ist die Einarbeitung in das Seminarthema soweit fortgeschritten, dass die Teilnehmer nun in der Lage sind, sich für ein besonderes Thema zu entscheiden. Dazu bilden sie Kleingruppen von 2 bis 3 Studenten möglichst aus verschiedenen Universitäten und Ländern. Das Organisationsteam und die lokalen Koordinatoren helfen bei der Themenfindung und Teamkoordination. Die ausgewählten Interessensgebiete werden dann in der jeweiligen Gruppe gemeinsam studiert und diskutiert, wobei chat-board und email verwendet werden. Die Teams informieren sich gegenseitig durch ,Status Reports" über ihre Aktivitäten. Das Organisationsteam und die lokalen Koordinatoren unterstützen die Teams gezielt bei der Vortragsplanung. Das Resultat der gemeinsamen Arbeit soll dann in Phase 3 vorgestellt werden.

Phase 3 (eine Woche im Juni) In der letzten Phase des Seminars stellen die Kleingruppen aus Phase 2 ihre Spezialthemen während eines gemeinsamen Workshops im Heinrich Fabri- Institut in Blaubeuren vor. Die studentischen Vorträge werden durch Übersichtsvorträge der lokalen Koordinatoren inhaltlich verbunden und ergänzt. Die Vorträge werden diskutiert und Interessierte zum weiteren wissenschaftlichen Arbeiten angeleitet. Hauptziel dieses abschließenden Treffens ist es, den Studenten Gelegenheit zu geben, Erfahrungen auf internationaler Ebene zu machen und nachhaltige wissenschaftliche Kontakte aufzubauen. Dadurch gelingt es auch, sonst sehr isolierte, kleine Interessensgruppen zu wissenschaftlichem Austausch zusammenzuführen.
The experience in Blaubeuren was very positive, both from human and scientific point of view. In particular, I find it very useful and interesting that young people can give a talk in a very relaxed atmosphere, where collaboration and discussions are easier than in other scientific meetings or seminars.

(Dr. Valentina Casarino, Torino)

Der Workshop zum dritten Internetseminar fand vom 18. bis 23. Juni 2000 statt. Das wissenschaftliche Programm bestand aus 35 Vorträgen von studentischen Teilnehmern und 5 Übersichtsvorträgen lokaler Betreuer. Die Teilnehmer kamen aus 16 Universitäten aus allen Kontinenten. Die am häufigsten vertretenen Länder waren (neben Deutschland) Italien, Marokko, Tschechien und die USA, aber auch Studenten aus Australien und Japan nahmen teil.

\section{Fazit}

Den Erfolg der Internetseminare und insbesondere die hohe Teilnehmerzahl am jeweiligen Workshop führen wir auf das Konzept einer Mischung aus Internetkommunikation und klassischem Seminar zurück. Lokale Seminargruppen unter fachlicher Betreuung werden in ein überregionales Team eingebunden, und Mathematik wird als internationale Wissenschaft erlebt. Während des Seminars wechseln sich für die Teilnehmer Lernen und Wissenserwerb kontinuierlich mit der Diskussion und Präsentation des Erlernten ab. Sie knüpfen erste internationale fachliche und persönliche Kontakte, wobei sie Englisch (und das Internet) als Basis moderner wissenschaftlicher Kommunikation erlernen und erfahren. Außerdem bietet das Internetseminar eine gute Möglichkeit, während eines Auslandsaufenthalts studienrelevante Leistungen an der Heimatuniversität zu erbringen.

I was delighted to see the group of students so enthusiastic about mathematics and so eager to study. I think that the participants of this program benefited a lot in both mathematical and social aspects.

(Prof. Yuri Latushkin, Missouri-Columbia)

Der Workshop ist zentraler Bestandteil des Internetseminars und nach unseren Erfahrungen die wesentliche Motivationsquelle für die teilnehmenden Studenten. Dies wird insbesondere durch die vielen Teilnehmer aus der ganzen Welt und die hohe Qualität der Vorträge bestätigt. Gleichwohl bedarf der Workshop einer sehr sorgfältigen Vorbereitung via Internetkommunikation. Es müssen Unterschiede im mathematischen Vorwissen ausgeglichen, eine gemeinsame inhaltliche Grundlage geschaffen und 
ein Gruppengefühl erzeugt werden. Hierfür hat sich das Programm BSCW in Verbindung mit dem chatboard bewährt. Die volle Integration als Seminar im Lehrplanangebot vieler Universitäten und die aus dem Seminar entstandenen nachhaltigen Forschungskontakte sind Anzeichen dafür, daß es auch oder gerade in der Mathematik an der Zeit ist, durch den Einsatz von Multimedia das traditionelle Unterrichtsangebot zu erweitern.

\section{Organisatorischer Rahmen}

Das Internetseminar Mathematik wird im Rahmen des Programms Virtuelle Hochschule BadenWürttemberg gefördert und ist Teil von Virtugrade - Virtuelle Graduiertenausbildung an der Universität Tübingen.

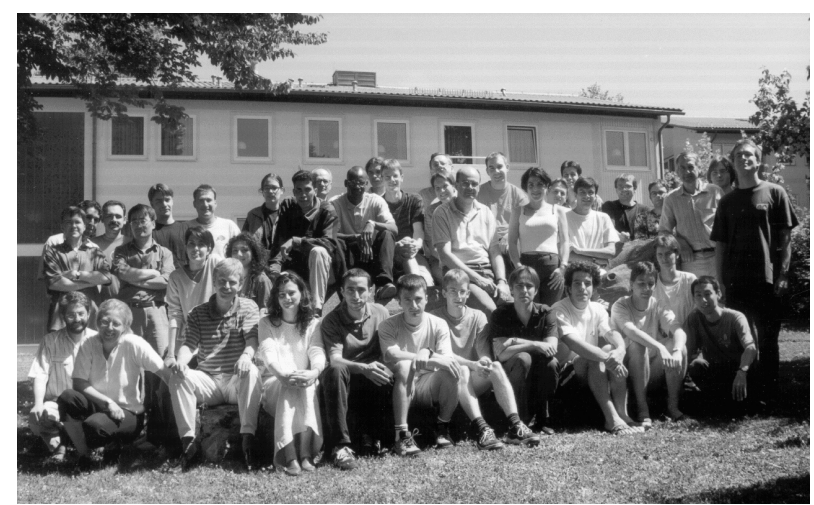

Weitere Informationen:

http://www . virtuelle-hochschule.de http://ww . virtugrade. uni-tuebingen. de http://michelangelo.mathematik.uni-tuebingen.de/ tulka/isem/index.html

\section{Anmerkung zur Anmerkung}

Wiederholung des relevanten Satzes aus der Anmerkung des Präsidenten (Mitteilungen der DMV 2-2000, S. 59): „Die Ausführungen von Herrn Pietsch stimmen insofern nicht, als sich unter den vorgeschlagenen Kandidaten zwei aus Universitäten in den neuen Bundesländern befinden (J. Kramer (HU Berlin), V. Mehrmann (TU Chemnitz))."

Ein Ossi oder „Neubundesbürger“ ist ein Mensch, der vor dem 3. Oktober 1990 einen Personalausweis der DDR hatte.

Ich habe behauptet, dass keiner der 28 Fachgutachter-Kandidaten zu der oben definierten Menge gehört. Deshalb weise ich die Unterstellung, dass meine Ausführungen nicht stimmen, entschieden zurück. Bekanntlich lässt sich jede Aussage über die NichtExistenz von Nullstellen einer Funktion durch eine Erweiterung des Definitionsbereiches falsch machen. Unter Mathematikern ist es allerdings üblich, die veränderten Voraussetzungen anzugeben und nicht das Wörtchen ,insofern“ zu bemühen.

Eine Schlussfolgerung, die ich in meinem „Offenen Brief" bewusst vermieden hatte, drängt sich nun zwangsläufig auf: ehemalige DDR-Bürger gelten (glücklicherweise nicht bei allen) als unerwünscht, wenn es darum geht, die neuen Bundesländer im Fachausschuss Mathematik der DFG zu vertreten.
Selbstverständlich dürfen auch die zugewanderten Kollegen nicht ausgegrenzt werden. Auf die richtige Mischung kommt es an, und dazu ist das von mir angemahnte Fingerspitzengefühl nötig.

Prof. Albrecht Pietsch

Mathematisches Institut

Friedrich-Schiller-Universität 07740 Jena

\section{Aus dem Protokoll der Präsidiumssitzung der DMV am 27. Mai 2000:}

Es wird noch einmal über den Leserbrief von Herrn Pietsch an die „Mitteilungen“ diskutiert. Man ist übereinstimmend der Meinung, dass die Nominierung der DFG-Kandidaten besser hätte organisiert werden können und vereinbart, beim nächsten Mal sorgfältiger vorzugehen. 